\title{
Modelos de regressão para estimação do volume de árvores comerciais, em florestas de Paragominas ${ }^{1}$
}

\author{
Eliana Nobre da Silva², Antônio Cordeiro de Santana ${ }^{3}$ \\ http://dx.doi.org/10.1590/0034-737X201461050005
}

\section{RESUMO}

Em fevereiro de 2009, o Ministério do Meio Ambiente criou a Resolução CONAMA 406, que determina que, a partir do segundo Plano Operacional Anual (POA), só será aceito, pelo órgão ambiental competente, o cálculo do volume de árvores em pé, mediante equação de volume, desenvolvida especificamente para o Plano de Manejo Florestal Sustentável (PMFS). Em função disso, necessita-se especificar um modelo estatístico para estimar o volume comercial das árvores. Neste trabalho, ajustou-se o modelo estatístico às informações coletadas em povoamento de floresta Ombrófila densa, localizada no município de Paragominas, Estado do Pará, com o objetivo de selecionar o de melhor ajuste e precisão. A amostra foi composta de 234 árvores, distribuídas em sete classes diamétricas, com amplitudes de $12 \mathrm{~cm}$, no intervalo de 49 até $127 \mathrm{~cm}$. Obteve-se o volume real, medindo-se as circunferências nas secções, a cada $2 \mathrm{~m}$, determinando-se o CAP e a altura comercial real. A seleção para a escolha do melhor modelo teve como referência o valor da estatística F, o mais alto coeficiente de determinação $\left(R^{2}\right)$, o menor erro padrão da estimativa em percentagem (Syx\%) e a análise de resíduos, por meio do Desvio Médio Porcentual (DMP). Com base nas estatísticas mencionadas, concluiu-se que o melhor entre os modelos tradicionais, a ser empregado na área, foi o proposto por Schumacher-Hall. Finalmente, concluiu-se que o modelo alternativo, com variável dummy, é melhor e deve ser o adotado em florestas nativas da Amazônia.

Palavras-chave: equações de volume, floresta Ombrófila, manejo florestal.

\section{ABSTRACT}

\section{Regression models for estimating the volume of commercial trees in forests in Paragominas}

In February 2009, the Ministryof the Environmentcreated theCONAMAResolution406, which provides thatfrom the secondAnnual Operating Plan(POA), thecalculation of volume ofstanding treeswill only beaccepted by thecompetent environmentalagencybythe volume equationdeveloped specifically for theSustainableForest Management Plan(PMFS). Thus, thestatistical modelto estimate the volumeofcommercialtrees needs to be specified. This study a djusted the statistical modelto the information collectedin a stand ofdenseOmbrophylousForestin Paragominas, Pará Stateto selectthe best fitand accuracy. The sample was composedof 234trees, distributed into sevendiameter class, with amplitudes of $12 \mathrm{~cm}$ in the range from $49 \mathrm{to} 127 \mathrm{~cm}$. The real volume was achieved by measuring the circumference in the sectionsat every $2 \mathrm{~m}$, determining the CBHandthe real tradeheight. The selectionfor choosing thebest model was based on the value of the F statistic, the highestcoefficient of determination $\left(\mathrm{R}^{2}\right)$, the loweststandard errorof estimate inpercentage(Syx \%) and the analysisof waste through thePercent Average Deviation (PAD). Based on the statisticsreferred to, it was concluded that the best traditional modelto beemployed inthe areawas the oneproposed bySchumacher-Hall. Finally, it is concluded that the alternative model with dummy variable is the best and should be adopted in the native forests of the Amazon.

Key words:equations ofvolume,forest management, Ombrophylous forest.

Recebido para publicação em 18/06/2013 e aprovado em 26/06/2014

${ }^{1}$ Trabalho extraído da Dissertação de Mestrado da primeira autora. Apoio financeiro: Conselho Nacional de Desenvolvimento Científico e Tecnológico.

${ }^{2}$ Engenheira Florestal, Mestre. Universidade Federal Rural da Amazônia, Avenida Presidente Tancredo Neves, 2501, Montese, 66.077-901, Belém, Pará, Brasil. eliana_nobre@ ig.com.br ${ }^{3}$ Engenheiro-Agrônomo, Doutor. Universidade Federal Rural da Amazônia, Avenida Presidente Tancredo Neves, 2501, Montese, 66.077-901, Belém, Pará, Brasil. acsantana@@ superig.com.br 


\section{INTRODUÇÃO}

O Pará é o principal produtor de madeira da Amazônia, com $45 \%$ do total produzido, concentra $51 \%$ das indústrias madeireiras e gera $48 \%$ dos empregos do setor, na Amazônia (Lentini et al., 2010). Em 2010, o Pará produziu $5.763 .823 \mathrm{~m}^{3}$ de madeira, em toras, caracterizando-se como o principal produtor (Brasil, 2010).

Em 2008, o setor florestal apresentou um valor adicionado de US\$ 4,46 bilhões e 30.481 empregos, participando, respectivamente com 9,6 e 3,6\% do PIB e do emprego total do Pará (Santana et al., 2010). Essa atividade internaliza grande parte da renda e do emprego no local onde se estabelece e é, portanto, um setor estratégico para o desenvolvimento rural da Amazônia. Os multiplicadores de emprego e de renda são respectivamente de 1,15 e 1,38 (Santana, 2009).

O manejo de florestas naturais e sua conservação demandam conhecimentos sobre sua estrutura e estoques correntes, bem como sobre os fatores que determinam sua evolução ao longo dos anos. Para tanto, é necessária a execução de inventários florestais, os quais, frequentemente, utilizam métodos e processos de amostragem com a finalidade de gerar informações sobre uma floresta natural ou plantada. Para a obtenção dos dados necessários à estimação dos modelos estatísticos, utilizou-se uma amostra da população. Os inventários florestais são instrumentos básicos, utilizados para se avaliar estatisticamente as reais potencialidades e capacidades produtivas dos recursos florestais de determinada área. São importantes ferramentas utilizadas no diagnóstico do potencial produtivo de florestas. É por meio dos resultados dos inventários florestais que se apoiam decisões acerca da viabilidade de empreendimentos florestais que exigem grandes investimentos $(\mathrm{Cu}-$ nha, 2004). O sucesso do inventário florestal está ligado à definição correta do processo de amostragem, do tamanho e forma das unidades amostrais e da intensidade de amostragem, requisitos básicos para se obterem as informações com precisão (Ubialli et al., 2009).

Com a escassez dos recursos florestais, em áreas nativas, e a consequente redução da biodiversidade, tornase cada vez mais importante melhorar os procedimentos de qualificação e avaliação dos produtos florestais madeireiros. Na presença de grandes estoques de florestas, compradores e vendedores não tendem a exigir rigor nas medidas das quantidades de madeira. A redução do estoque de madeira proveniente de florestas nativas causou elevação dos preços de madeira, em toras e, como consequência, aumentou a exigência sobre o controle do dimensionamento e da qualidade dos produtos. Em função disso, aumentou o controle sobre a medição de produtos e subprodutos florestais, no processo de comerciali- zação. Com isso, necessita-se de dados quali-quantitativos sobre as florestas, envolvendo informações sobre o volume de madeira por espécie e por hectare, estoque de regeneração natural, dentre outros (Cunha, 2004).

Neste contexto, o objetivo deste trabalho foi testar modelos estatísticos de dupla entrada, visando a estimar, com maior precisão, os volumes individuais das espécies florestais de terra firme, no município de Paragominas, mediante a utilização das variáveis independentes diâmetro e altura.

\section{MATERIAL E MÉTODOS}

Os dados, utilizados para a estimação dos parâmetros das equações de volume, foram obtidos de árvores abatidas, na exploração de uma área de 95,91 ha. A área de estudo localiza-se na unidade de produção anual (UPA $\mathrm{N}^{\circ}$ 11) e na unidade de trabalho (UT N ${ }^{\circ}$ 4), da fazenda Rio Capim, que possui uma área de 149.190,54 ha, localizada no município de Paragominas.

O ambiente fitoecológico do município é representado pela Floresta Ombrófila densa, de elevada ocorrência na região amazônica (Martins Filho, 2006).

O clima do município é do tipo AW, ou seja, tropical chuvoso, com estação seca bem definida, segundo Köppen. As temperaturas médias do ar variam de 25,6 a $27,8{ }^{\circ} \mathrm{C}$, caracterizando temperaturas médias elevadas durante todo o ano. A precipitação pluviométrica, na região tropical é o elemento meteorológico de maior variabilidade climática. Os totais pluviométricos anuais variam de 857,8 a 2.787,7 $\mathrm{mm}$ e os principais solos, na região do Município de Paragominas, são Latossolos Amarelos, Argissolos Amarelos, Plintossolos, Gleissolos e Neossolos (Rodrigues et al., 2003).

Foram cubadas 234 árvores-amostra e, em cada árvore derrubada, foi determinado o volume real, $\mathrm{em}^{3}$, por meio da fórmula utilizada por Silva et al. (1984), medindo-se, para tanto, a circunferência das secções, a cada $2 \mathrm{~m}$, e determinando-se a circunferência, a altura do peito, em $\mathrm{cm}$ (CAP), e a sua altura comercial real, em (m), sendo mensuradas a $1,30 \mathrm{~m}$ do solo e a $30 \mathrm{~cm}$ acima das sapopemas, quando estas ocorriam (Tabela 1).

A seleção das espécies foi feita de acordo com os interesses econômicos da empresa, por ocasião da exploração florestal. Para cada árvore-amostra derrubada, foi determinado o volume real, que conta com a medida da circunferência, nas secções de 2 em 2 m, com o DAP e com a altura comercial real.

$\mathrm{Na}$ área de estudo, em um período de 24 meses, houve coletas de dados de todos os indivíduos, mensurados por meio das variáveis diâmetro a altura do peito - DAP $(\mathrm{cm})$ e altura do fuste / árvore (m). O diâmetro à altura do peito (DAP) foi coletado com fita métrica, ou seja, 
mede-se, na verdade, a "Circunferência à Altura do Peito" (CAP), a 1,30 m do solo, para, posteriormente, ser convertida em DAP. A relação entre DAP e CAP foi determinada pela fórmula abaixo:

$\mathrm{DAP}=\frac{\mathrm{CAP}}{\pi}$

A altura comercial foi considerada da base do corte $(0,15 \mathrm{~cm}$ do solo $)$ até o ponto onde houve a remoção das copas das árvores derrubadas, e o volume real de cada árvore foi obtido mediante o emprego da fórmula utilizada por Silva et al. (1984), conforme equação abaixo:

$v=\sum \frac{g_{i}+g_{i+1}}{2} \cdot l_{i}$

em que $v=$ volume total; $g_{i}=$ área basal na i-ésima posição; $l_{i}=$ comprimento da secção na i-ésima posição.

A seleção do modelo de regressão mais adequado foi baseada nas seguintes estatísticas: mais alto coeficiente de determinação $\left(\mathrm{R}^{2}\right)$; menor erro padrão de estimativa $\left(\mathrm{S}_{\mathrm{yx}}\right)$; menor coeficiente de variação $(\mathrm{CV})$; análise de resíduos, por meio do Desvio Médio Percentual (DMP), para verificar a não tendenciosidade na distribuição dos resíduos e o percentual de subestimativa ou superestimativa dos volumes, pelos modelos; valor de $\mathrm{F}$ altamente significante e o teste de White para verificar a presença de heteroscedasticidade dos erros de cada regressão.

$\mathrm{Na}$ estimação do volume, inicialmente foram considerados os oito modelos de equações de regressão, amplamente divulgados na literatura (Tabela 2).

Também foram analisados os problemas de multicolinearidade nos modelos de dupla entrada, por meio do fator de variância inflacionária, que é determinado a partir do coeficiente de determinação, obtido das regressões auxiliares, especificadas com base nas variáveis independentes, conforme descrito em Santana (2003).

Nas florestas nativas da Amazônia, é comum encontrar muitos valores atípicos (Santos et al., 2011) e sua retirada da amostra tende a enviesar os resultados. Também, a não consideração desses outliers no modelo pode produzir erro de especificação do modelo. Para contornar o problema, apresenta-se o modelo 09 , com variável dummy, para captar os efeitos dos valores atípicos, para mais e para menos, dos dados. $\ln V_{i}=a+b_{1} \ln D_{i}=b_{2} \ln H_{i}+b_{3} V d a_{i}+b_{4} V d b_{i}+\varepsilon_{i}$ em que Vda é a variável dummy, que capta os valores atípicos muito altos e, Vdb, os valores atípicos muito baixos.

\section{RESULTADOS E DISCUSSÃO}

Os resultados referem-se aos parâmetros estatísticos obtidos pelo ajuste das equações de volume, com casca, a partir de dados da cubagem real. Os resultados revelaram que os modelos ajustados apresentaram significância, a 1\%. Os valores dos coeficientes de determinação $\left(R^{2}\right)$, variando de 94 a $96 \%$, indicam um alto grau de ajuste da variável dependente (volume) às variáveis independentes (DAP e altura).

O coeficiente de variação (CV\%) ficou entre 10,47, para o modelo 08 , e $15,44 \%$, para o modelo 01 , representando baixa variabilidade dos dados e boa capacidade para extrapolação dos resultados a partir deles. Com relação à estatística "F", que atesta a existência do modelo de regressão, obtiveram-se todos os valores significativos, a $1 \%$ de probabilidade de erro. O Desvio Médio Percentual (DMP), por sua vez, variou entre $-1,32 \%$, para o modelo 05 e $0,61 \%$, para o modelo 08 .

Além dessas informações, levou-se em consideração a significância da estatística t, de Student, associada às estimativas dos parâmetros. Essa estatística é fundamental porque um valor não diferente de zero indica que a variável não é relevante para explicar as variações do volume, e, conforme Santana (2003), altos valores das estatísticas $\mathrm{R}^{2}$ e $\mathrm{F}$ e estatísticas t não significantes são sintomas de elevado grau de multicolinearidade nas regressões múltiplas. $\mathrm{O}$ teste de variância inflacionária demonstrou presença de forte multicolinearidade, nos modelos de dupla entrada, com exceção dos modelos 07 e 09.

Com base nesses critérios, os modelos que apresentaram todas as estatísticas t diferentes de zero e ausência de multicolinearidade e de heteroscedasticidade foram o modelo Logarítmico, de Schumacher e Hall (modelo 07), e o modelo 09, com variável dummy, sendo o eleito para fazer as estimativas dos volumes.

Tabela1 - Distribuição do número de indivíduos por classe de diâmetro das árvores-amostra

\begin{tabular}{lccc}
\hline Classes de diâmetro $(\mathbf{c m})$ & Diâmetro $(\mathbf{c m})$ & Centro de classes $(\mathbf{c m})$ & $\mathbf{N}^{\mathbf{o}}$ de indivíduo \\
\hline 1 & $49+61$ & 55 & 84 \\
2 & $61+73$ & 67 & 86 \\
3 & $73+85$ & 79 & 42 \\
4 & $85+97$ & 91 & 10 \\
5 & $97+109$ & 103 & 10 \\
6 & $109+121$ & 115 & 1 \\
7 & $121+133$ & 127 & 1 \\
\hline Total & & & 234 \\
\hline
\end{tabular}


Com relação a outros estudos, utilizando-se equação de volume em diferentes regiões do Brasil, na região noroeste de Mato Grosso, a equação de SH apresentou maior precisão para estimar o volume, em função do diâmetro e da altura total das árvores (Colpini et al., 2009). Na região de Barbalha, Ceará, o modelo logarítmico de $\mathrm{SH}$, para calcular os volumes, considerando-se seções de 1,0 m de comprimento, e volume da primeira tora como variável independente, Silva et al. (1993) concluíram que o modelo volumétrico proposto por $\mathrm{SH}$ foi o mais apropriado, para o caso estudado.

Em um estudo para Eucalyptus grandis, no Vale do Rio Doce, em Minas Gerais, trabalhando com o modelo logarítmico de SH, Guimarães \& Leite (1996) concluíram que equações de volume, ajustadas a partir de 150 árvores, forneceram estimativas volumétricas similares às obtidas pelo emprego da equação resultante da cubagem de 500 árvores. Também em Minas Gerais, em uma pesquisa sobre análise de equações volumétricas para Eucalyptus spp., segundo o método de regeneração, na região de José de Melo, onde foram considerados sete estratos, sendo o primeiro um plantio em regime de alto fuste e, os demais estratos, brotações, em regime de primeira talhadia. Paula Neto et al. (1983) consideram mais apropriado, para os casos estudados, o modelo volumétrico de SH. De maneira semelhante, estudos baseados em dados coletados de floresta nativa, realizados por Silva et al. (1984) e Silva \& Carvalho (1984), para a FLONA Tapajós e Belterra, respectivamente, apontaram que o modelo mais conveniente para predizer os volumes individuais das árvores foi o modelo de SH.
Pelo que se observa, em todos os estudos prévios, independentemente da área de floresta ser nativa ou plantada, o modelo SH foi o que apresentou melhor adequação. Na verdade, a distribuição amostral dos dados de volume de madeira tende a apresentar uma curva assimétrica para a direita. Neste caso, a aplicação de transformação logarítmica aos dados tende a corrigir esse problema e, por conseguinte, essa transformação logarítmica contribui para eliminar o problema de heteroscedasticidade nos resíduos das regressões estimadas, uma vez que permite a uniformização dos dados (Santana, 2003).

O teste para homoscedasticidade de White foi realizado por meio do software Eviews 6, cujos resultados indicaram a rejeição da hipótese de presença de heteroscedasticidade dos erros da regressão, no modelo de SH, o que indica que a variância dos erros é constante, para todas as observações (Tabela 3). Portanto, os três testes apresentados aceitam a hipótese nula de homoscedasticidade dos erros da regressão.

Diante desse resultado, assume-se que as estimativas dos parâmetros associados às variáveis DAP e H são não viesadas e eficientes, conforme Santana (2003). Assim, pode-se interpretar os resultados da equação proposta por SH, apresentados na Tabela 4.

Os resultados mostram que a regressão é válida, pois a estatística $\mathrm{F}$ foi significativa, a $1 \%$. O coeficiente de determinação, ajustado por graus de liberdade, mostra que $94,11 \%$ das variações do volume são explicadas pelas variáveis DAP e H. Todas as estatísticas $t$ apresentaram significância a 1\%. Também, a estatística de DurbinWatson, que testa autocorrelação de primeira ordem nos

Tabela 2 - Equações volumétricas usadas para a estimativa do volume com casca de 28 espécies florestais, Fazenda Rio Capim, Paragominas, PA

\begin{tabular}{lll}
\hline $\mathbf{N}^{\mathbf{0}}$ & Equações & Autor \\
\hline 01 & $\mathrm{~V}=\beta_{0}+\beta_{1} \mathrm{~d}^{2} \mathrm{~h}+\varepsilon$ & Spurr \\
02 & $\mathrm{~V}=\beta_{0}+\beta_{1} \mathrm{~d}^{2}+\beta_{2} \mathrm{~d}^{2} \mathrm{~h}+\beta_{3} \mathrm{~h}+\varepsilon$ & Stoate \\
03 & $\mathrm{v}=\beta_{0}+\beta_{1} \mathrm{~d}^{2}+\beta_{2} \mathrm{~d}^{2} \mathrm{~h}+\beta_{3} \mathrm{dh} \mathrm{h}^{2}+\beta_{4} \mathrm{~h}^{2}+\varepsilon$ & Nãslund \\
04 & $\mathrm{~V}=\beta_{0}+\beta_{1} \mathrm{~d}+\beta_{2} \mathrm{~d}^{2}+\beta_{3} \mathrm{dh}+\beta_{4} \mathrm{~d}^{2} \mathrm{~h}+\beta_{5} \mathrm{~h}+\varepsilon$ & Meyer \\
05 & $\mathrm{~V}=\beta_{0}+\beta_{1} \mathrm{~d}+\beta_{2} \mathrm{~d}^{2}+\beta_{3} \mathrm{dh}+\beta_{4} \mathrm{~d}^{2} \mathrm{~h}+\varepsilon$ & Meyer Modificada \\
06 & $\mathrm{Lnv}=\beta_{0}+\beta_{1} \ln \left(\mathrm{d}^{2} \mathrm{~h}\right)+\varepsilon$ & Logarítmico de Spurr \\
07 & $\operatorname{Lnv}=\beta_{0}+\beta_{1} \ln (\mathrm{d})+\beta_{2} \ln (\mathrm{h})+\varepsilon$ & Logarítmico de Schumacher e Hall \\
08 & $\operatorname{Lnv}=\beta_{0}+\beta_{1} \ln (\mathrm{d})+\beta_{2} \ln \left(\mathrm{d}^{2}\right)+\beta_{3} \ln (\mathrm{h})+\beta_{4} \ln \left(\mathrm{h}^{2}\right)+\varepsilon$ & Logarítmico de Prodan \\
\hline
\end{tabular}

Em que: $v=$ volume; $d=$ diâmetro à altura do peito; $h=$ altura comercial; $\beta_{0}, \beta_{1}, \beta_{2}, \beta_{3}, \beta_{4}, \beta_{5}=$ coeficientes; $\ln =\log$ aritmo neperiano. Fonte: Silva et al. (1984) e Thomas et al. (2006).

Tabela 3 - Resultados do teste de White para heteroscedasticidade

\begin{tabular}{llll}
\hline Teste de Heteroscedasticidade: White & & & \\
\hline Estatística F & 0.847895 & Prob. F(5,228) & 0.5171 \\
Obs*R-quadrado & 4.271615 & Prob. Chi-Square(5) & 0.5110 \\
Scaled explained SS & 6.085785 & Prob. Chi-Square(5) & 0.2980 \\
\hline
\end{tabular}

Rev. Ceres, Viçosa, v. 61, n.5, p. 631-636, set/out, 2014 
resíduos, indica que não são autocorrelacionados contemporaneamente. Portanto, as estimativas são não viesadas e eficientes (Tabela 4).

Com relação à análise das estimativas dos parâmetros, tem-se aqui uma novidade, pois em nenhum artigo foi encontrada a análise e interpretação desses resultados. Assim, as estimativas dos parâmetros associados a cada variável independente refletem elasticidades, ou seja, mostram a mudança proporcional, no volume, para cada alteração porcentual na respectiva variável.

Portanto, para cada variação de $1 \%$ no diâmetro das árvores, tem-se uma variação de 2,01\% no volume de madeira, mantendo-se a influência da altura fixa. Igualmente, a cada aumento de $1 \%$ na altura da árvore, temse um incremento de $0,88 \%$ no volume.
Os resultados do modelo com variável dummy estão na Tabela 5. Observa-se que os coeficientes das variáveis dummies foram significantes, a 1\%, o que atesta que os valores atípicos altos e baixos são deslocadores da equação de volume. Assim, a não consideração desses efeitos produz estimativas errôneas para o volume das árvores.

Por fim, os resultados mostram que o modelo com variável dummy é adequadamente especificado e a não incorporação das informações dos valores atípicos torna-o modelo com erro de especificação. Como, na floresta amazônica, esse fenômeno está presente, o modelo com variável dummy deve ser adotado, em substituição aos demais modelos até então utilizados para a estimação volumétrica das árvores.

Tabela 4 - Resultados do modelo proposto por SH, estimado no Eviews 6

\begin{tabular}{|c|c|c|c|c|}
\hline Variável & Coeficiente & Desvio Padrão & Estatística - $\mathbf{t}$ & Probabilidade \\
\hline $\mathrm{C}$ & $-9,508421$ & 0,179833 & $-52,87361$ & 0,0000 \\
\hline $\operatorname{Ln}(\mathrm{D})$ & 2,013940 & 0,039194 & 51,38402 & 0,0000 \\
\hline $\operatorname{Ln}(\mathrm{H})$ & 0,878799 & 0,032881 & 26,72688 & 0,0000 \\
\hline R-quadrado & 0,941702 & \multicolumn{2}{|c|}{ Mean dependent var } & 1,464954 \\
\hline R-quadrado ajustado & 0,941197 & \multicolumn{2}{|c|}{ S.D. dependent var } & 0,454742 \\
\hline S.E. da regressão & 0,110271 & \multicolumn{2}{|c|}{ Akaike info criterion } & $-1,559007$ \\
\hline Estatística - F & $1.865,706$ & \multicolumn{2}{|c|}{ Schwarz criterion } & $-1,514708$ \\
\hline Prob (F-statistic) & 0,000000 & \multicolumn{2}{|c|}{ Estatística Durbin-Watson } & 1,932903 \\
\hline
\end{tabular}

Tabela 5 - Resultados do modelo proposto com variável dummy, estimado no Eviews 6

\begin{tabular}{|c|c|c|c|c|}
\hline Variável & Coeficiente & Desvio Padrão & Estatística - t & Probabilidade \\
\hline $\mathrm{C}$ & -8.930925 & 0.219184 & -40.74628 & 0.0000 \\
\hline $\operatorname{Ln}(\mathrm{D})$ & 1.905115 & 0.047082 & 40.46376 & 0.0000 \\
\hline $\operatorname{Ln}(\mathrm{H})$ & 0.836789 & 0.032165 & 26.01590 & 0.0000 \\
\hline Vda & 0.099373 & 0.037485 & 2.650997 & 0.0086 \\
\hline $\mathrm{Vdb}$ & -0.225023 & 0.045791 & -4.914108 & 0.0000 \\
\hline R-quadrado & 0,94807 & \multicolumn{2}{|c|}{ Mean dependent var } & 1,464954 \\
\hline R-quadrado ajustado & 0,94716 & \multicolumn{2}{|c|}{ S.D. dependent var } & 0,454742 \\
\hline S.E. da regressão & 0,10245 & \multicolumn{2}{|c|}{ Akaike info criterion } & $-1,657508$ \\
\hline Estatística - F & $1.045,115$ & \multicolumn{2}{|c|}{ Schwarz criterion } & $-1,583676$ \\
\hline Prob(F-statistic) & 0,000000 & \multicolumn{2}{|c|}{ Estatística Durbin-Watson } & 2,071187 \\
\hline
\end{tabular}

\section{CONCLUSÕES}

Os modelos logarítmicos, em geral, apresentaram melhores ajustes que os modelos lineares, em função da distribuição assimétrica para a direita da amostra, que contribui para violar o pressuposto de homoscedasticidade da variância do termo de erro da equação.

O modelo escolhido não apresentou problemas de heteroscedasticidade e as estimativas dos parâmetros foram todas significativas a $1 \%$.
O modelo de Schumacher e Hall apresentou o melhor ajustamento dos dados, entre os modelos tradicionais. Em função disso, foi selecionado e recomendado para a estimação volumétrica das árvores comerciais da área de estudo.

O modelo com variável dummy mostrou-se adequado à estimação do volume das árvores amostradas, devendo substituir os demais modelos, que tendem a gerar valores errados para o volume das árvores.

Rev. Ceres, Viçosa, v. 61, n.5, p. 631-636, set/out, 2014 


\section{REFERÊNCIAS}

Brasil (2010) Polícia Militar Ambiental. Ecossistema: floresta amazônica. Disponível em: <http://www.pmambientalbrasil.org.br/ecos-flamazonica.htm>. Acessado em: 17 de junho de 2010.

Colpini C, Travagin DP, Soares TS \& Silva V S M (2009) Determinação do volume, do fator de forma e da porcentagem de casca de árvores individuais em uma Floresta Ombrófila Aberta na região noroeste de Mato Grosso. Acta Amazônica, 39:97-104.

Cunha US da (2004) Dendrometria e Inventario Florestal. Disponíve em: 〈http://www.obt.inpe.br/prodes/Relatorio_Prodes2008.pdf>. Acessado em: 27 de setembro de 2010.

Guimarães DP \& Leite HG (1996) Influência do número de árvores na determinação de equação volumétrica para Eucalyptus grandis. Revista scientia Florestalis, 50:37-42.

Lentini M, Veríssimo A \& Pereira D. (2010) A expansão madeireira na Amazônia. Disponível em: <http://www.imazon.org.br/novo2008/ publicacoes_ler.php?idpub=120>. Acessado em: 08 de fevereiro de 2010.

Martins Filho SEC (2006) Avaliação dos danos e métodos de regulação da floresta submetida à exploração de impacto reduzido na Amazônia Oriental. Dissertação de Mestrado. Universidade Federal Rural da Amazônia, Belém. 71p.

Paula Neto F de, Souza AL de, Quintaes P CG \& Soares VP (1983) Análise de equações volumétricas para Eucalyptus spp., segundo o método de regeneração na região de José de Melo em Minas Gerais. Revista Árvore, 7:56-70.

Rodrigues TE, Silva R das C, Silva JML da, Oliveira Júnior RG de, Gama RJNF \& Valente MA (2003) Caracterização e classificação dos solos do município de Paragominas, Estado do Pará. Belém, EMBRAPA. 51p. (Série Documentos, 162).
Santana AC de (2009) Análise das contas correntes do balanço de pagamentos interestadual do estado do Pará, período 2005 a 2008. Belém, UFRA. 50p.

Santana AC de, Santos MAS \& Oliveira CM (2010) Comportamento histórico da produção e comércio de madeira do estado do Pará e nos mercados local e internacional. Amazônia: Ciência \& Desenvolvimento, 6:63-90.

Santana AC de (2003) Métodos quantitativos em economia: elementos e aplicações. Belém, UFRA. 484p.

Santos PC, Santana AC de, Barros PLC, Queiroz JCB \& Vieira TO (2011) O emprego da geoestatística na determinação do tamanho "ótimo" de amostras aleatórias com vistas à obtenção de estimativas dos volumes dos fustes de espécies florestais em Paragominas, estado do Pará. Acta Amazônica, 41:213-222.

Silva JAA da, Meunier IMJ, Borders BE, Farias GG de A \& Assunção EP (1993) Equação volumétrica para Eucalyptus camaldlensis, na região de Barbalha, Ceará, usando o volume da primeira tora como variável independente. Revista Árvore, 17:30-37.

Silva JNM, Carvalho JOP de, Lopes J do CA \& Carvalho MSP de (1984) Equações de volume para a Floresta Nacional do Tapajós. Boletim de Pesquisa Florestal, 8:50-63.

Silva JNM \& Carvalho MSP de (1984) Equações de volume para uma floresta secundária no planalto do Tapajós-Belterra, PA. Boletim de Pesquisa Florestal, 8:1-15.

Thomas C, Andrade CM, Schneider PR \& Finger CAG (2006) Comparação de equações volumétricas ajustadas com dados de cubagem e análise de tronco. Ciência Florestal, 16:319-327.

Ubialli JA, Afonso Filho F, Machado S do A \& Arce JE (2009) Comparação de métodos e processos de amostragem para estimar a área basal para grupos de espécies em uma floresta ecotonal da região norte matogrossense. Acta Amazônica, 39:305-314. 